\title{
Psychophysical and Physiological Evidence for Parallel Afferent Pathways Mediating the Sensation of Itch
}

\author{
Lisa M. Johanek, ${ }^{1}$ Richard A. Meyer, ${ }^{1,3}$ Tim Hartke, ${ }^{1}$ Joseph Greg Hobelmann, ${ }^{2}$ David N. Maine, ${ }^{2}$ Robert H. LaMotte, ${ }^{4}$ \\ and Matthias Ringkamp ${ }^{1}$ \\ Departments of ${ }^{1}$ Neurosurgery and ${ }^{2}$ Anesthesiology and ${ }^{3}$ The Applied Physics Laboratory, Johns Hopkins University, Baltimore, Maryland 21287 , and \\ ${ }^{4}$ Department of Anesthesiology, Yale, New Haven, Connecticut 06520
}

The neuronal pathways for itch have been characterized mainly based on responses to histamine. Intracutaneous application of histamine produces intense itch and a large area of axon-reflexive vasodilation ("flare") around the application site. Both phenomena are thought to be mediated through neuronal activity in itch-specific, mechanoinsensitive C-fiber afferents (CMi). However, mechanical and electrical stimuli that do not activate CMi fibers can cause the sensation of itch, and itch may occur without flare, suggesting that other neuronal itch pathways exist. Because cutaneous application of spicules from the plant Mucuna pruriens (cowhage) has been anecdotally reported to produce itch without flare, we performed psychophysical experiments to investigate whether the mechanisms underlying cowhage- and histamine-induced itch differ. Although histamine and cowhage produced itch of similar magnitude, the itch to cowhage was not correlated with the itch to histamine; some subjects had intense itch to cowhage and little itch to histamine and visa versa. Laser Doppler measurements of blood flow revealed that histamine led to a large area of vasodilation, whereas cowhage produced vasodilation restricted to the application site. Pretreatment of the skin with an antihistamine blocked the itch produced by histamine but did not prevent cowhage-induced itch. Desensitization of the skin with topical capsaicin abolished cowhage-induced itch but did not significantly alter histamine-induced itch. These findings indicate that cowhage itch is signaled through a population of capsaicin-sensitive afferent nerve fibers that is distinct from CMi fibers mediating histamine-induced itch. Cowhage may be useful to investigate the neural pathway mediating nonhistaminergic itch.

Key words: pruritus; nociceptors; cowhage; capsaicin; antihistamine; flare

\section{Introduction}

Many chronic itch conditions of dermatological, neurological, and other (systemic) origin are resistant to antihistamine treatment (Twycross et al., 2003), providing a strong rationale for exploring nonhistaminergic mechanisms of itch. Our present understanding of the neuronal mechanisms underlying the sensation of itch, however, has been mainly obtained in experiments that used histamine as the itch-producing (pruritic) stimulus. In addition to the sensation of itch, histamine administration into human skin produces a large area of vasodilation (flare) around the application site (Simone et al., 1987; Magerl et al., 1990) that is of neurogenic origin and is mediated via an axon reflex (Lynn, 1996).

In microneurography experiments in human, iontophoresis of histamine only weakly activated C-polymodal fibers (Handwerker et al., 1991; Schmelz et al., 1997). The cutaneous receptive

Received March 20, 2007; revised June 4, 2007; accepted June 4, 2007.

This work was supported by National Institutes of Health Grant P01 NS 4739, with additional support from the Johns Hopkins Blaustein Pain Research Fund. Dr. Pathak (Zandu Pharmaceuticals, Mumbai, India) generously donated the cowhage pods used in these studies. We thank Cheryl Carmona and Drs. Lisa Beck, James Campbell, and Srinivasa Raja for their input and technical advice in these studies.

Correspondence should be addressed to Matthias Ringkamp, Department of Neurosurgery, School of Medicine, Johns Hopkins University, Meyer 5-109, 600 North Wolfe Street, Baltimore, MD 21287. E-mail: platelet@jhmi.edu. DOI:10.1523/JNEUROSCI.1249-07.2007

Copyright $\odot 2007$ Society for Neuroscience $\quad$ 0270-6474/07/277490-08\$15.00/0 field size of these afferents in man is, furthermore, too small $(\sim 1$ $\mathrm{cm}^{2}$ ) (Schmidt et al., 1997) to account for the large area of histamine-induced flare. Therefore, activity in mechano-heatsensitive $\mathrm{C}$ fibers $(\mathrm{CMH})$ is unlikely to mediate the itch and flare induced by histamine iontophoresis. In contrast to $\mathrm{CMH}$ fibers, a subpopulation of mechanoinsensitive C-fiber afferents (CMi) in human was vigorously excited by histamine, and the neuronal activity in histamine-sensitive CMi fibers paralleled the time course of the itch sensation (Schmelz et al., 1997). CMi fibers branch extensively in the skin, and their receptive field size is approximately five times that of $\mathrm{CMH}$ fiber afferents (Schmidt et al., 2002). These findings suggest that histamine-induced itch and flare are mediated through a subpopulation of itch-specific CMi fibers (Schmelz et al., 1997). Centrally, these afferents are thought to project onto specific lamina I neurons in the dorsal horn, providing a correlate for a central pathway for the sensation of itch (Andrew and Craig, 2001). However, it is unlikely that the sensation of itch is mediated exclusively through CMi fibers, because itch can be produced by mechanical (von Frey, 1922; Shelley and Arthur, 1957), punctate heat (Shelley and Arthur, 1957), and electrical stimuli (Ikoma et al., 2005) that are unlikely to activate CMi fibers. In addition, electrical stimuli can cause itch in the absence of a flare response (Ikoma et al., 2005). These observations suggest that a population of afferent fibers other than CMi fibers can mediate the sensation of itch. 
Spicules from the plant Mucuna pruriens (cowhage) have been used previously to study the neuronal mechanism of itch (Shelley and Arthur, 1957; Tuckett and Wei, 1987). Anecdotal observations reported that cowhage-induced itch may be accompanied by only a small, local skin reddening (Graham et al., 1951; Shelley and Arthur, 1955b), suggesting that the mechanisms of cowhageand histamine-induced itch differ. To gain insights into the neural mechanisms of cowhage-induced itch, we investigated the flare response from cowhage and histamine, the dependence of cowhage induced itch on histaminergic mechanisms, and the role of capsaicin-sensitive nerve fibers in cowhage- induced itch.

\section{Materials and Methods}

Volunteers for the studies were recruited through flyers posted at Johns Hopkins University. They were informed about the goal of the study and the procedures involved. Subjects underwent a physical examination, and only healthy volunteers without allergies or antihistamine treatment were allowed to participate. The subjects gave written consent and were free to withdraw from the experiment at any time. Subjects were compensated for participation in the study. Studies were approved by the Institutional Review Board committee at Johns Hopkins University. Administration of cowhage was approved by the Federal Drug Administration (FDA) as an investigational new drug.

An experimental session of the different protocols described in detail below lasted $\sim 90 \mathrm{~min}$. All subjects tolerated the application of the different stimuli and interventions well. No unexpected side effects were observed after the application of cowhage spicules or the intracutaneous injection of histamine.

Study 1: cowhage- and histamine-induced itch and flare. The aim of this study was to investigate the magnitude of itch sensation and the changes in skin blood flow produced by topical application of cowhage spicules and intradermal injection of histamine. Twelve healthy volunteers were involved in the study (age range, 22-63 years; average \pm SD, $38.8 \pm 13.7$ years; seven male and five female).

There were four application sites, two on each forearm. The proximal and distal test sites on each volar forearm were $4.5 \mathrm{~cm}$ from the center of the forearm. Subjects received each of the following stimuli: histamine, saline, and active and inactive cowhage spicules. Applications were randomized with the restriction that the combinations of histamine/saline and active/inactive cowhage were always applied to either the proximal or distal test sites. The two distal applications were always performed first, followed by the two proximal applications. Subjects rated the itch to each substance using a magnitude estimation technique (see below). Laser Doppler images were used to measure vasodilation (see below).

Study 2: effect of antihistamine on cowhage-induced itch. This study was performed to investigate the involvement of histaminergic mechanisms in cowhage-induced itch. A topical antihistamine cream or a placebo cream was administered before application of histamine or cowhage in 15 healthy volunteers (age range, 20-59 years; average \pm SD age, $31 \pm 10$ years; six male and nine female). Experiments were conducted in two experimental sessions separated by 2 weeks.

The day before the experimental session, a $2 \mathrm{~cm}^{2}$ box was marked at a site $4.5 \mathrm{~cm}$ proximal and $4.5 \mathrm{~cm}$ distal to the center of the volar forearm. These four sites (two on each forearm) served as the pretreatment sites. Application sites for the antihistamine cream (Zonalon; Bioglan Pharmaceuticals Company, Malvern, PA) and placebo cream (Professional Arts Pharmacy, Windsor Mill, MD) were randomized with the exception that, in a given subject, both proximal and both distal sites always received the same treatment. On the first day of treatment, subjects were told to apply the creams at home according to instructions on the Zonalon package, i.e., four times a day. Zonalon contains doxepine hydrochloride (5\%) as the active ingredient, which blocks $\mathrm{H}_{1}$ and $\mathrm{H}_{2}$ receptors (van der Goot and Timmerman, 2000) and has been shown previously to inhibit histamine-induced itch after topical application (Bernstein et al., 1981). Treatment sites remained the same for the two experimental sessions.

On the day of the experiment, subjects were instructed to apply the cream at least once before coming to the laboratory. When the subject arrived, a final cream application was performed, and the experiment began 15 min later. In one session, the subject received two active cowhage and two inactive cowhage applications (one of each at the antihistamine-treated site, one of each at the placebo-treated site). In the other session, the subject received two histamine and two saline injections (one of each at the antihistamine-treated site, one of each at the placebo-treated site). The order of the two sessions was randomized. In each session, the order of the different stimuli was randomized and testing alternated between arms. Subjects rated the itch to each substance using a magnitude estimation technique (see below). Laser Doppler images were used to measure vasodilation (see below).

Study 3: involvement of capsaicin-sensitive fibers in cowhage-induced itch. Pretreatment of the skin with capsaicin was used to investigate the role of capsaicin-sensitive nerve fibers in cowhage- and histamine-induced itch. Six healthy volunteers were involved in the study (age range, $24-60$ years; average \pm SD, $35.3 \pm 14.1$ years; three male and three female). The experimental sessions took place on 2 different days.

Each volar forearm was pretreated for 3 consecutive days with either $0.1 \%$ capsaicin cream (Capzaicin HP; Chattem, Chattanooga, TN) or a placebo cream (Vanicream; Pharmaceutical Specialties, Rochester, MN) under an occlusive dressing. A similar protocol has been used previously at the thigh, where it resulted in an almost complete loss of epidermal nerve fibers (Polydefkis et al., 2004). An adhesive $10 \times 4 \mathrm{~cm}$ patch $(1 \mathrm{~mm}$ thick; 3 M Foam Medical Tape, $3 \mathrm{M}$, St. Paul, MN) with an $8 \times 2 \mathrm{~cm}$ center cutout was placed on each volar forearm, forming a shallow well into which creams were placed. The well on the nondominant arm was filled with $\sim 2 \mathrm{~g}$ of capsaicin cream, whereas the well on the dominant arm was filled with $\sim 2 \mathrm{~g}$ of a placebo cream. The patches were covered with an adhesive Tegaderm I.V. dressing (3M) to form an occlusive dressing that was kept on overnight. The patches and cream were replaced daily by the experimenter. On the morning of the fourth day, the patches were removed, and the $8 \times 2 \mathrm{~cm}$ application areas were outlined on the subject's forearm. To let capsaicin-induced signs of hyperalgesia subside, the first test session was performed on the day after removal of the patches. The second session always took place on a different day but was conducted within $6 \mathrm{~d}$ after the removal of the patches. In one session, the subject received two active cowhage and two inactive cowhage applications (one each at the capsaicin-treated site, one each at the placebo-treated site). In another session, the subject received two histamine and two saline injections (one each at the capsaicin-treated site, one each at the placebotreated site). The order of the two sessions was randomized. In addition, in each session the order of the different stimuli was randomized and testing alternated between arms.

Cutaneous heat testing with the blunt end of a $49^{\circ} \mathrm{C}$ brass rod was performed preceding each experimental session to determine both the degree of and maximal areas of desensitization. Two areas within the capsaicin-treated skin where subjects reported only pressure or warm sensation (versus hot or pain sensation on the contralateral arm) were marked with a felt-tipped pen for application of the itch-producing substances. Two areas were also marked within the placebo-treated site. Subjects rated the itch to each substance using a magnitude estimation technique (see below).

Pruritic stimuli. An identical dose of histamine was used in the three studies. Histatrol ( $1 \mu \mathrm{g}$ of histamine phosphate in $10 \mu \mathrm{l}$; ALK-Abello, Round Rock, TX) was administered by intradermal injection using 28 gauge needle syringes (Lo-Dose; Becton Dickinson, Franklin Lakes, NJ). Injection of an equal amount of sterile saline served as control. Intradermal injections were used instead of iontophoresis, because a device approved by the FDA for the iontophoresis of histamine was not commercially available. For the application of cowhage spicules (Zandu Pharmaceuticals, Mumbai, India), small pieces of pods with (active, inactive) cowhage spicules were pressed against the skin (application area, $1 \mathrm{~cm}^{2}$ ), resulting in 18 spicules on average being inserted into the skin. Pods with active cowhage spicules had been sterilized by irradiation, whereas autoclaving was used to produce sterile and inactive spicules. The appearance of the active and inactivated pod pieces was not different.

The time interval between the application of different stimuli was 20 
min to allow all sensations from a stimulus to vanish before a new stimulus was applied. In each study, the stimulus sequence was randomized. However, to avoid order effects, randomization was balanced such that each stimulus appeared at each position of the sequence with similar frequency.

Itch ratings. In all studies, a magnitude estimation technique was used to rate the magnitude of itch sensation (Stevens and Galanter, 1957; Meyer and Campbell, 1981). Subjects were trained on the principles of magnitude estimation and then were asked to assign an arbitrary number (modulus) to their recollection of the magnitude of itch associated with a mosquito bite. During the study, subjects verbally rated their itch sensation relative to this modulus every $10 \mathrm{~s}$ for $10 \mathrm{~min}$.

Laser Doppler scans. A scanning laser Doppler system (Moor Instruments, Devon, UK) was used to measure cutaneous blood flow over an area of $6 \times 10 \mathrm{~cm}(80 \times 132$ pixels resolution, 4 $\mathrm{ms} /$ pixel scan time) around the stimulus application site. The system produces color-coded images that are proportional to the blood cell perfusion (or flux). The scanner head was always set at a distance $33 \mathrm{~cm}$ from the midline of the arm. Two baseline laser Doppler scans of cutaneous blood flow were taken before any stimulus application. Subsequent scans were obtained starting at 2 and 9 min after administration of each substance. Because the scans took $\sim 1.5 \mathrm{~min}$ to complete, we refer to the scans as being at " 3 min" and " 10 min." After the final scan, the area of visible flare was marked on the subject's forearm and traced onto a sheet of transparency paper. Room temperature was maintained at $\sim 23^{\circ} \mathrm{C}$.

Blinding. Inactive control substances were used in each study to blind the experimenter and the volunteer. In study 2 , both the experimenter and participant were also blind to the antihistamine/placebo treatment site. Blinding of capsaicin/placebo was not performed in study 3 so that the capsaicin could be applied to the participant's nondominant arm. In addition, the pain at the capsaicin treatment area and the heat testing after the pretreatment revealed the capsaicin-treated area.

Image analysis. Because we were interested in changes in blood flow associated with the application of the pruritic agents, laser Doppler flare images were analyzed by averaging the two baseline scans and subtracting this average from all images. This procedure removed most of the background signal, although some images still had residual flow artifacts attributable to slight movement of the arm between scans; these artifacts were mainly associated with blood vessels. To eliminate the majority of this background noise, images then underwent a threshold function to eliminate pixels falling under 125 perfusion units (PU). The number of pixels with $>125$ perfusion units was counted and used to estimate the area of flare $\left(1\right.$ pixel $\left.=0.57 \mathrm{~mm}^{2}\right)$.

Visual flare area was determined by cutting the flare shape out of the transparency paper and weighing the paper on a semi-micro balance. A 1 $\mathrm{cm}^{2}$ piece of transparency paper was used as a standard.

Statistical analysis. To analyze data across subjects, each itch rating from a subject was normalized by dividing by the rating that subject had assigned to the modulus (mosquito bite). To assess itch over time, itch ratings were averaged over two time intervals: $0-3.5$ and 6.5-10 min. These were termed "interval 1" and "interval 2," respectively, and ap- proximate the time of the laser Doppler scans (see Fig. 1). Because data across subjects were not normally distributed, we presented the results as medians \pm 25 th and 75 th quartiles. Friedman's ANOVA with Dunn's multiple comparisons were performed when appropriate.

\section{Results}

Study 1: cowhage- and histamine-induced itch and flare

Topical application of cowhage spicules and intradermal injection of histamine produced itch in all 12 subjects tested. Saline and inactive cowhage did not produce itch. Figure $1 A$ displays the itch ratings obtained from a typical subject over the $10 \mathrm{~min}$ recording period. The peak magnitude of itch was similar for both histamine and cowhage; however, the time course of itch differed between the two substances. The cowhage itch dissipated during the $10 \mathrm{~min}$ recording, whereas the histamine itch did not decrease substantially.

Average itch ratings over interval 1 and interval 2 were calculated to obtain a single value of itch at two time points for each 


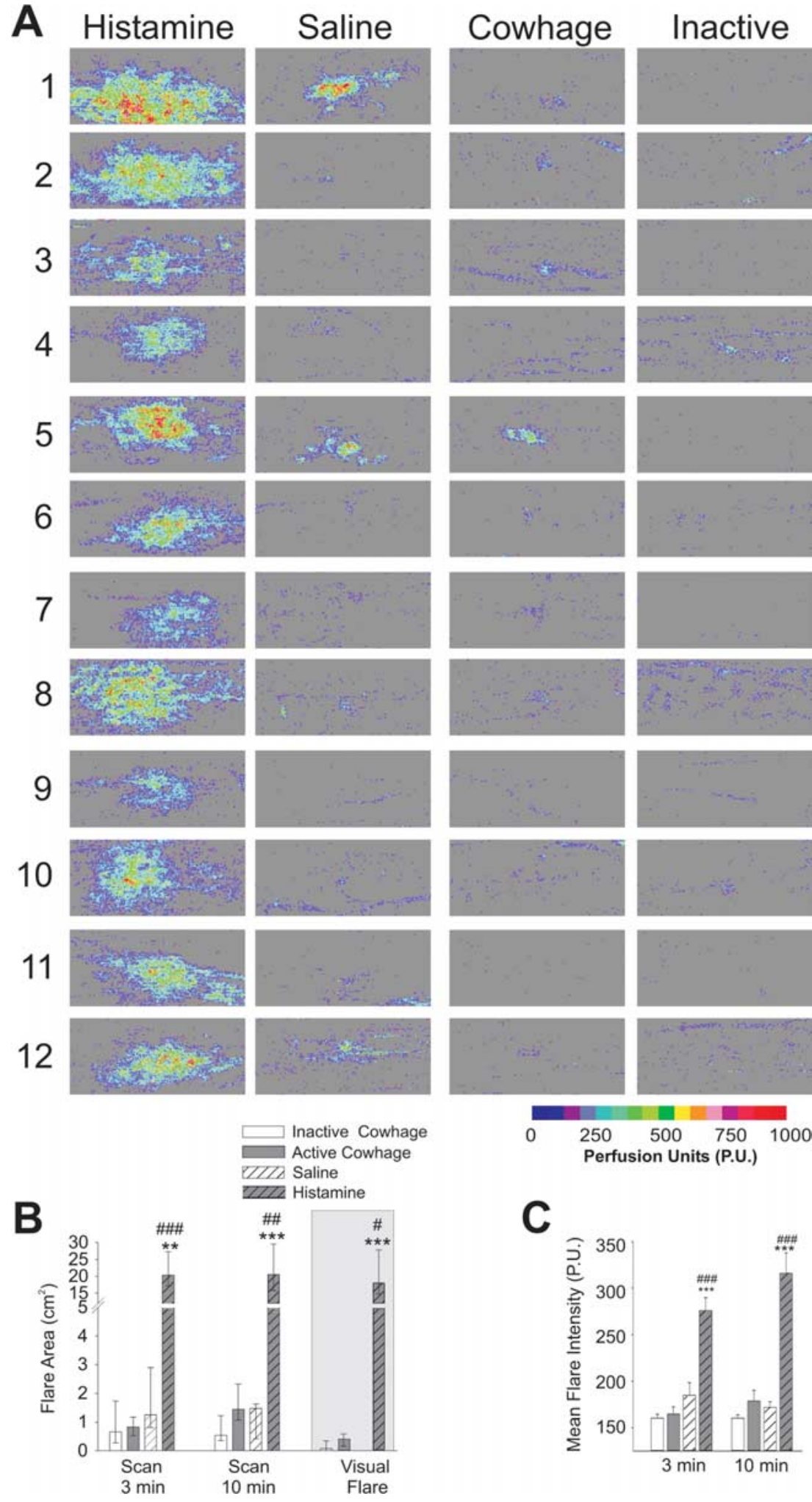

Figure 2. Histamine but not cowhage produced a large area of flare. The area and the intensity of flare after histamine administration was significantly greater than the area and intensity of flare after active cowhage, inactive cowhage, and saline. $\boldsymbol{A}$, Analyzed laser Doppler images from each subject (1-12) showing scans obtained 3 min after application of histamine, saline, cowhage, and inactive cowhage. Histamine-induced flare was substantial in each subject. Flares produced by saline, cowhage, and inactive cowhage were minimal. $\boldsymbol{B}$, The laser Doppler flare area was computed based on the number of "valid pixels" remaining after the threshold procedure was performed. Visual flare was marked on the skin 10 min after the drug applications (Friedman's repeated-measures ANOVA, $p<0.0001$, with Dunn's multiple comparisons; median area \pm 25 th and 75th quartiles). C, Mean flare intensity computed based on the number of pixels above an intensity of $125 \mathrm{PU}$ (repeated-measures ANOVA, $p<0.0001$, with Bonferroni's multiple comparisons; ${ }^{* *} p<0.01$ and ${ }^{* * *} p<0.001$, histamine vs saline and ${ }^{\#} p<0.05$, ${ }^{\# \#} p<$ 0.01 , and ${ }^{\# \# \#} p<0.001$, histamine vs active cowhage; mean \pm SEM). subject. These time periods were chosen to correspond with the laser Doppler flare images obtained at 3 and $10 \mathrm{~min}$. The itch produced by the active compounds (histamine and active cowhage) was always greater than the itch produced by the inactive compounds (saline and inactive cowhage) (Fig. $1 B, C$ ). Although the averaged itch evoked by cowhage did not differ from the averaged itch evoked by histamine for these two time points, cowhage-induced itch did tend to subside more rapidly than histamine-induced itch. Itch ratings to cowhage were less during interval 2 versus interval $1(p<0.01)$, whereas the itch ratings to histamine were not different for these two time periods.

Laser Doppler images of changes in cutaneous blood flow after administration of saline, histamine, active cowhage, and inactive cowhage are shown in Figure $2 \mathrm{~A}$. These images were obtained $3 \mathrm{~min}$ after application and are similar to those obtained at 10 min after application. Histamine reliably produced a substantial flare response, seen both in the laser Doppler scans and on visual examination of the skin. In contrast, the development of skin reddening after cowhage application was variable between subjects, and, when reddening did occur, it was much smaller than the histamine flare (Fig. $2 \mathrm{~A}$, subjects 3 and $5)$. The vasodilation produced by cowhage (when present) was always localized to the area of spicule application. Furthermore, a localized reddening also occurred on occasion after inactive cowhage application (Fig. $2 A$, subjects 4 and 10) and after saline injection (Fig. $2 A$, subjects 1 and 5). Areas of vasodilation determined by analysis of the laser Doppler images and by measurement of visual flare demonstrate that histamine-induced flare was larger than saline, inactive cowhage, and active cowhage vasodilation at 3 and $10 \mathrm{~min}$ after application (Fig. $2 B$ ).

The intensity of blood flow was also obtained from the laser Doppler scans. Histamine-induced flares were more intense than vasodilation produced by any other substance (Fig. 2C). Images generated after histamine injection had a larger mean pixel intensity than images after cowhage application at both the $3 \mathrm{~min}$ $(276 \pm 14$ vs $165 \pm 8$ PU; $p<0.001)$ and $10 \min (316 \pm 22$ vs $178 \pm 12$ PU; $p<$ 0.001) time points. Likewise, the maximum pixel intensity was greater for histamine than for cowhage at both time points ( 3 min, $912 \pm 44$ vs $354 \pm 41 \mathrm{PU}, p<$ 0.001; $10 \mathrm{~min}, 948 \pm 31$ vs $490 \pm 64 \mathrm{PU}$, $p<0.01)$. 
Study 2: effect of antihistamine on cowhage-induced itch

Pretreatment with the antihistamine successfully prevented the histamine-evoked itch (Fig. $3 A$ ), but the antihistamine had no effect on the cowhage-evoked itch. Average itch ratings over interval 1 were calculated to obtain a single value of total itch produced for each subject. Antihistamine pretreatment suppressed the itch generated after histamine injection but not after cowhage application (Fig. 3B).

In addition, the area of histamineinduced flare measured at $3 \mathrm{~min}$ after histamine injection was reduced by antihistamine pretreatment (Fig. 3C). The area of vasodilation induced by cowhage was similar to that seen in the first study, and there was no reduction in area by antihistamine pretreatment. Topical antihistamine pretreatment also reduced the histamineinduced flare area at the $10 \mathrm{~min}$ time point (data not shown).

Finally, we sought to determine whether the area of flare correlated with the magnitude of the itch sensation. Because activity in a subset of CMi fibers is thought to be responsible for both magnitude of histamine-induced itch and extent of flare, one would expect these two variables to be correlated. Histamine-induced itch and flare were correlated at both the placebo- and antihistamine-treated sites (Fig. 4A). Interestingly, although both histamine-induced itch and flare were reduced by the antihistamine pretreatment, the correlation between itch and flare persisted. In addition, the histamine-induced flare area at the placebo-treated site strongly correlated with the histamine-induced flare area at the antihistamine-treated site (Fig. 4C), suggesting that subjects with large flare responses still had larger flare responses after antihistamine pretreatment. There was no correlation between cowhage-induced itch and flare (Fig. $4 B$ ).

Although the magnitude of itch to histamine (and to cowhage) varied greatly across subjects (see large confidence intervals in Fig. $1 B$ ), the ratings to repeated applications of cowhage or histamine in a given subject were highly correlated (cowhage, $r=0.66, p<0.01$; histamine, $r=0.70, p<0.01$ ). Thus, subjects who gave a high (or a low) rating of itch to one application were likely to rate a subsequent application of the same pruritic agent as high (or low). In contrast, the ratings of itch to cowhage in a given subject were not correlated with the corresponding ratings of itch to histamine (Fig. 5). Subjects could have high levels of itch to cowhage and low ratings of itch to histamine and vise versa. Histamine- and cowhageinduced itch were not correlated in study 2, in which these substances were administered to placebo-treated sites on different test days, or in study 1 , in which substances were administered in the same test session. These data support the notion that the mechanisms of cowhage- and histamineinduced itch are independent.

\section{Study 3: involvement of capsaicin-sensitive fibers in cowhage-induced itch}

After capsaicin desensitization, all subjects displayed decreased heat sensation in the capsaicin-treated area. Cowhage-induced itch was virtually absent in the capsaicin-pretreated skin (Fig. 6). In contrast, histamine-induced itch was reduced by $\sim 30 \%$, but this change did not reach statistical significance. Thus, the superficial, capsaicin-sensitive nerve terminals that are desensitized by topical capsaicin play an important role in the itch produced by cowhage spicules that are inserted into the superficial layers of the skin.

\section{Discussion}

This study provides evidence for two distinct neuropathways for itch sensation. Our data suggest that histaminergic-itch and nonhistaminergic itch (induced by cowhage administration) are mediated through distinct classes of primary afferent nerve fibers. We find the following. (1) Histamine-induced, but not cowhageinduced, itch is accompanied by a large area of flare. (2) The magnitude of cowhage- and histamine-induced itch do not correlate. (3) Antihistamine significantly reduced histamineinduced itch but had no effect on cowhage itch. (4) Capsaicin desensitization abolished cowhage- induced itch but only slightly attenuated the itch produced by histamine.

Similar to a previous study (Simone et al., 1987), subjects 


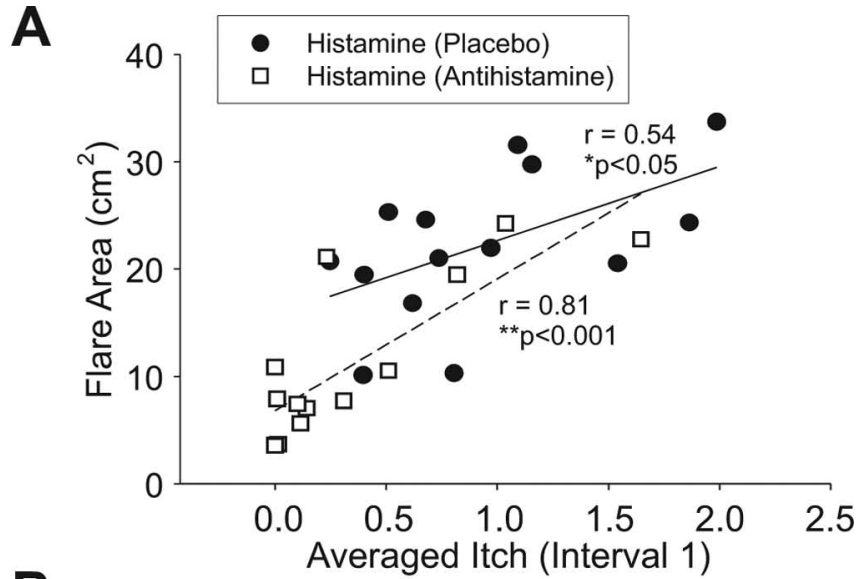

B
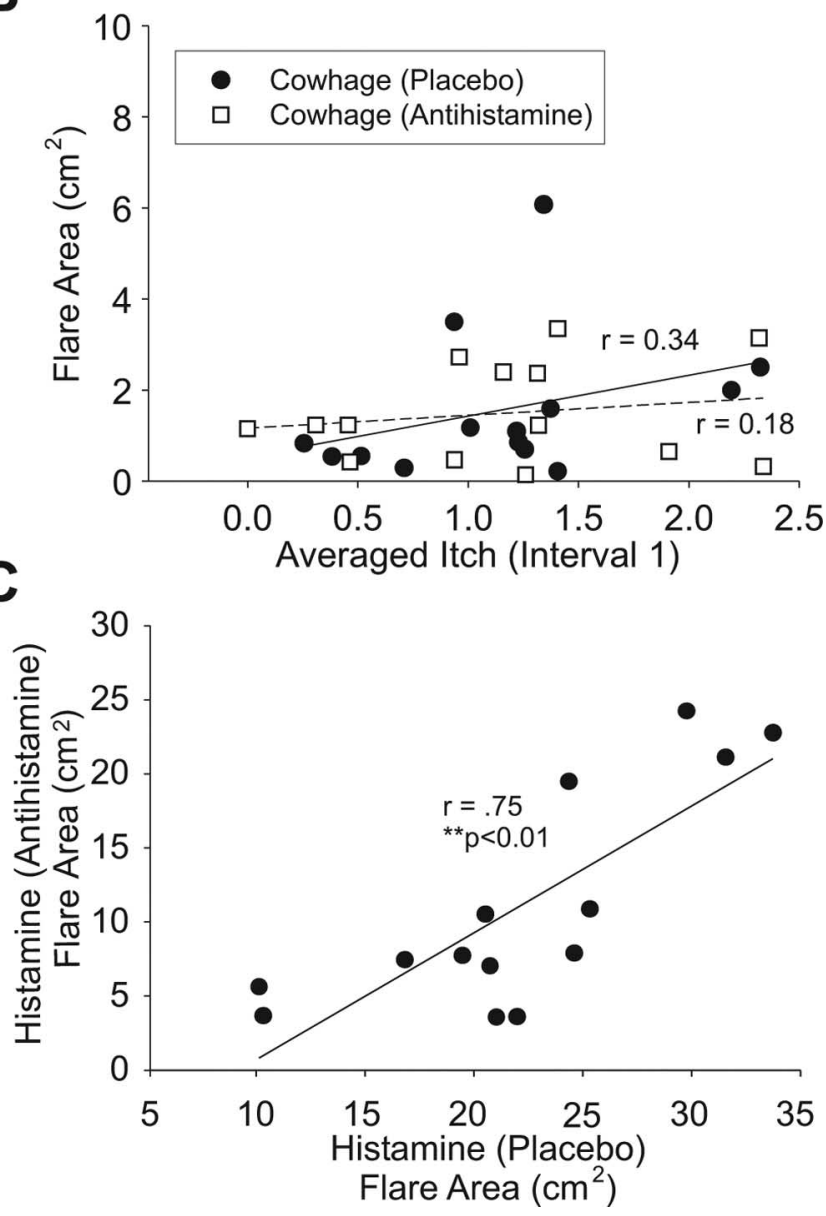

Figure 4. Histamine-induced flare is correlated with the magnitude of itch sensation. Cowhage-induced flare is not correlated with itch. The flare area determined from the laser Doppler scan is plotted as function of the averaged itch rating in interval 1 for each subject $(n=$ 14). $A$, Histamine flare area versus averaged itch rating $(0-3.5 \mathrm{~min}) . \boldsymbol{B}$, Cowhage flare area versus averages itch rating $(0-3.5 \mathrm{~min})$. Note different scale. $C$, Flare area induced by histamine at the antihistamine-treated site is correlated with the flare area at the placebo-treated site.

rated the intensity of their itch sensation based on their recollection of the itch produced by a mosquito bite. Despite the high variability of interindividual ratings, individual subjects were able to reliably scale itch from repeated cowhage and histamine administrations. Therefore, individual subjects were consistent in the rating of their itch sensation. Furthermore, the histamine stimulus used in this study produced itch of a magnitude similar to previous reports (Simone et al., 1987, 1991). Subjects were

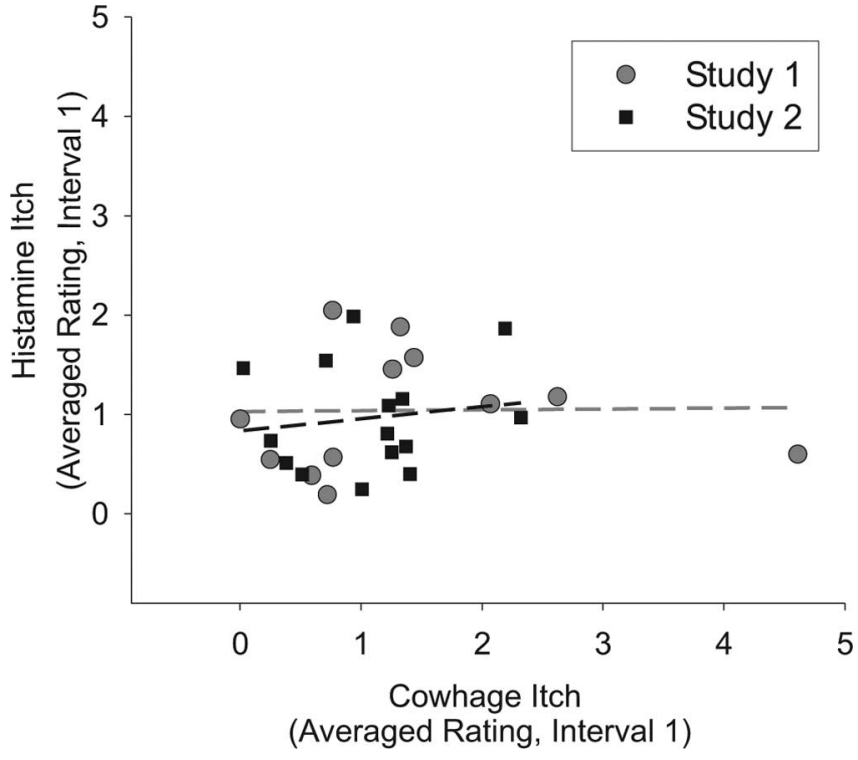

Figure 5. Cowhage-induced itch does not predict histamine-induced itch, suggesting independent mechanisms of action. For each subject, the averaged itch ratings (interval 1) for histamine are plotted against the average itch ratings for cowhage. Data come from study 1 (gray circles, gray dashed line) and the placebo site of study 2 (black squares, black dashed line).

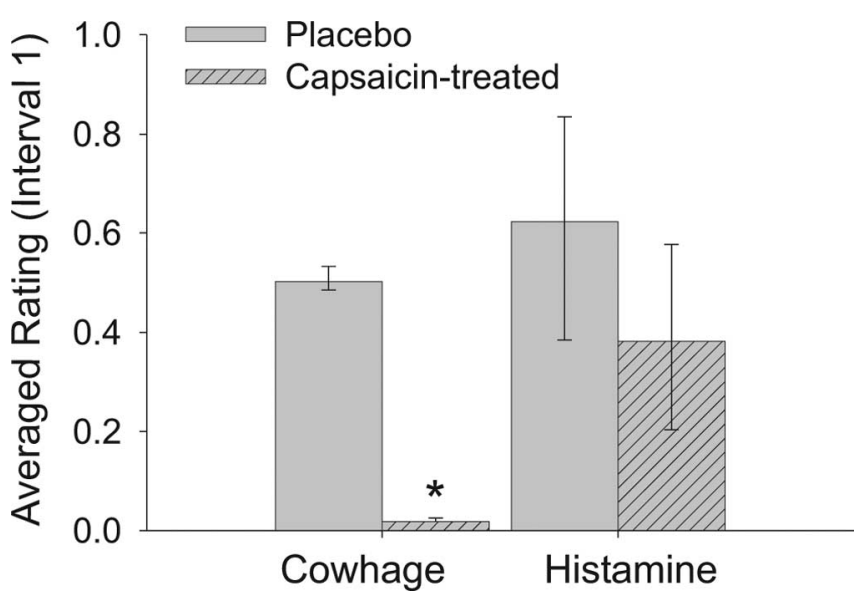

Figure 6. Capsaicin pretreatment prevented cowhage-induced itch. Subjects $(n=6)$ rated the intensity of itch sensation from cowhage and histamine at sites pretreated for $3 \mathrm{~d}$ with either a placebo cream or $0.1 \%$ capsaicin cream. Itch ratings were averaged over the $0-3.5 \mathrm{~min}$ time period (median \pm 25 th and 75 th quartiles; ${ }^{*} p<0.05$, Wilcoxon's matched-pairs test).

asked to focus on the sensation of itch caused by the different stimuli. In addition to itch, cowhage causes nociceptive sensations such as burning and pricking (Graham et al., 1951; Shelley and Arthur, 1955b). These sensations are, however, of lesser magnitude and duration than the itch sensation (Masood et al., 2005). Similar nociceptive sensations are also reported after the intradermal injection of histamine (R. H. LaMotte, unpublished observation).

\section{Lack of flare in cowhage-induced itch}

Although histamine and cowhage caused itch of similar magnitude, only histamine produced a large area of vasodilation. Several lines of evidence indicate that flare is mediated through CMi. (1) Stimulation of CMi, but not $\mathrm{CMH}$, nociceptors produced vasodilation in pig skin (Lynn, 1996). (2) Electrical stimulation of the skin at a strength known to excite $\mathrm{CMHs}$ did not produce a 
flare response; however, with a higher current density and recruitment of CMi fibers, a persistent flare was produced (Schmelz et al., 2000a). (3) Capsaicin produced a large flare response, and the activity in CMi fibers closely matched the duration of capsaicin-induced burning pain (Schmelz et al., 2000b). (4) The innervation territories of CMi fibers (Schmidt et al., 2002) are large enough to account for the extensive capsaicin and histamine flares.

For histamine, we observed a correlation between itch intensity and flare area. Previous studies have also found a modest correlation between itch intensity and flare or blood flow after histamine application (Magerl and Handwerker, 1988; Darsow et al., 1996). This would be expected if CMi fibers were responsible for histamine-induced itch and flare.

When cowhage did induce vasodilation, it remained localized to the application area. In addition, the flare area did not differ between saline and active and inactive cowhage. Therefore, cowhage-induced vasodilation is likely attributable to localized skin irritation rather than an axon reflex. These findings substantiate previous anecdotal observations that cowhage produces intense itch without flare (Graham et al., 1951; Shelley and Arthur, 1955b).

Because a flare was absent, cowhage likely did not activate CMi fibers. The higher transcutaneous electrical activation threshold for CMi fibers (Weidner et al., 1999) suggests that they may terminate in deeper skin layers. These deeper layers were likely not reached by the cowhage spicules. Injection of the active cowhage ingredient into deeper cutaneous tissues may activate CMi fibers. However, the absence of a flare after superficial spicule insertion demonstrates that activation of CMi fibers is not necessary to produce intense itch. Our findings are in agreement with a previous study showing that electrical stimulation at intensities insufficient to activate CMi fibers was able to produce the sensation of itch without flare (Ikoma et al., 2005).

\section{Lack of correlation between cowhage- and histamine-induced itch} If cowhage and histamine itch were mediated through the same nerve fibers, the magnitude of sensation should correlate. However, such a correlation was not observed. Because our application method for cowhage did not allow precise control over the number, depth, or orientation of inserted spicules, the lack of correlation between the ratings of cowhage- and histamineinduced itch could be attributable to the variability of the cowhage stimulus. This, however, is unlikely, because we observed a strong correlation between the itch ratings for repetitive cowhage (or histamine) applications. Because the application methods of cowhage spicules (skin prick) and histamine (intradermal injection) were quite different, mainly superficial afferents were likely activated by cowhage, whereas histamine excited afferents in deeper skin layers. The lack of correlation between the magnitude of cowhage- and histamine-induced itch provides additional evidence that the itch sensation to these stimuli is mediated through separate afferent channels.

\section{Cowhage-induced itch is nonhistaminergic}

One possible explanation for the production of cowhage-induced itch is the activation of histamine-releasing mast cells. A previous study on the effect of the $\mathrm{H}_{1}$ blocker mepyramine on cowhage itch was inconclusive because the majority of subjects ( 9 of 13) still reported intense itch after cowhage application, although the effect on histamine itch was not tested (Broadbent, 1953). In the present study, topical antihistamine treatment did not affect cowhage itch, whereas histamine itch was abolished. Failure of the antihistamine to inhibit cowhage-induced itch is unlikely attributable to insufficient pretreatment. If cowhage does cause release of histamine from mast cells, the amount is likely to be $<1$ $\mu \mathrm{g}$ because injection of $1 \mu \mathrm{g}$ of histamine produced a large flare, whereas cowhage did not. However, antihistamine treatment significantly inhibited flare and itch induced by this dose of histamine. We conclude that cowhage itch does not invoke a histaminergic mechanism.

Previous studies suggested that the pruritogen in cowhage is a proteinase, mucunain (Shelley and Arthur, 1955a,b). However, the exact mechanisms by which cowhage produces the sensation of itch are currently unknown. The endopeptidases kallikrein and papain also produce itch through nonhistaminergic mechanisms and without a flare response (Shelley and Arthur, 1955b; Hagermark et al., 1972; Hagermark, 1973, 1974). Interestingly, the proteinase-activated receptor-2 (PAR-2) and an upregulation of tryptase have both been implicated in causing pruritus in patients with atopic dermatitis (Steinhoff et al., 2003). One possibility is that cowhage acts via activation of PAR-2.

\section{Capsaicin-sensitive nerve fibers mediate \\ cowhage-induced itch}

Capsaicin desensitization abolished cowhage itch but did not significantly affect histamine-induced itch. Previous studies described a reduction in histamine-induced flare and itch after capsaicin pretreatment (Handwerker et al., 1986; Toth-Kasa et al., 1986; Weisshaar et al., 1998). However, the magnitude of decrease depended on the number of capsaicin applications and the elapsed time after capsaicin treatment (Toth-Kasa et al., 1986). Furthermore, topical application of capsaicin cream did not eliminate itch sensation after histamine iontophoresis but reduced its magnitude by $\sim 30 \%$ (Weisshaar et al., 1998), which is comparable with the decrease we observed. Although the number of subjects in our capsaicin study may have been too small to detect a significant reduction of histamine itch, our results clearly indicate that cowhage- and histamine-induced itch can be dissociated.

The smaller effect of capsaicin on histamine- versus cowhageinduce itch could be attributable to an inadequate capsaicin treatment of deeper skin layers. Topical application of capsaicin under an occlusive dressing for $2 \mathrm{~d}$ leads to almost complete loss of epidermal nerve fibers (Polydefkis et al., 2004). Despite the fact that we extended this treatment to $3 \mathrm{~d}$, capsaicin pretreatment reduced but failed to significantly affect histamine itch, although cowhage-induced itch was absent.

Alternatively, the smaller effect of capsaicin on histamine itch may indicate that each form of itch is mediated through neuronal activity in different sets of afferent nerve fibers. The afferents that terminate in deeper layers of the skin (possibly histaminesensitive CMi fibers) may be less capsaicin sensitive than the superficial epidermal nerve fibers that mediate cowhage-induced itch.

\section{Conclusion}

We provide evidence for at least two independent populations of primary afferents mediating the sensation of itch. One population mediates histaminergic itch and consists of CMi fibers (Schmelz et al., 1997). The other population signals nonhistaminergic itch (e.g., produced by cowhage) and consists of superficial capsaicin-sensitive afferents. Because cowhage spicules activate polymodal C-fibers in cat (Tuckett and Wei, 1987), monkey (L. M. Johanek, unpublished observations), and humans (B. Namer, unpublished observations), these afferents are likely part of this population. How pruriceptive information from these two types of peripheral sensory neurons is processed centrally is 
only beginning to be revealed. In cat, spinothalamic tract neurons have been found that have selective input from afferents similar to CMi fibers in humans (Andrew and Craig, 2001). Such central neurons have yet to be identified in the primate. Conversely, capsaicin-responsive, polymodal spinothalamic tract dorsal horn neurons have been identified in monkeys that are selectively responsive to either cowhage or histamine (Davidson et al., 2005). Because both primary afferent and spinothalamic neurons responsive to cowhage or to histamine also respond to nociceptive stimuli, their precise role in mediating pruritic and nociceptive sensory qualities evoked by histamine and cowhage needs additional study.

Chronic itch is often resistant to antihistamine treatment. Additional studies of the nonhistaminergic pathway activated by cowhage spicules might provide insights into the neural basis for this itch.

\section{References}

Andrew D, Craig AD (2001) Spinothalamic lamina I neurons selectively sensitive to histamine: a central neural pathway for itch. Nat Neurosci 4:72-77.

Bernstein JE, Whitney DH, Soltani K (1981) Inhibition of histamineinduced pruritus by topical tricyclic antidepressants. J Am Acad Dermatol 5:582-585.

Broadbent JL (1953) Observations on itching produced by cowhage, and on the part played by histamine as a mediator of the itch sensation. Br J Pharmacol 8:263-290.

Darsow U, Ring J, Scharein E, Bromm B (1996) Correlations between histamine-induced wheal, flare and itch. Arch Dermatol Res 288:436-441.

Davidson S, Zhang X, Khasabov SG, Simone DA, Giesler GJ (2005) Characterization of pruriceptive spinothalamic tract neurons in the monkey. Soc Neurosci Abstr 31:171.20.

Graham DT, Goodell H, Wolff HG (1951) Neural mechanisms involved in itch, "itchy skin", and tickle sensations. J Clin Invest 30:37-49.

Hagermark O (1973) Influence of antihistamines, sedatives, and aspirin on experimental itch. Acta Derm Venereol (Stockh) 53:363-368.

Hagermark O (1974) Studies on experimental itch induced by kallikrein and bradykinin. Acta Derm Venereol (Stockh) 54:397-400.

Hagermark O, Rajka G, Bergvist U (1972) Experimental itch in human skin elicited by rat mast cell chymase. Acta Derm Venereol (Stockh) $52: 125-128$

Handwerker HO, Magerl W, Klemm F, Lang E, Westerman RA (1986) Quantitative evaluation of itch sensation. In: Fine afferent nerve fibers and pain (Schmidt RF, Schaible HG, Vahle-Hinz C, eds), pp 461-473. Weinheim, Germany: VCH Verlagsgemeinschaft.

Handwerker HO, Forster C, Kirchhoff C (1991) Discharge patterns of human C-fibers induced by itching and burning stimuli. J Neurophysiol 66:307-315

Ikoma A, Handwerker H, Miyachi Y, Schmelz M (2005) Electrically evoked itch in humans. Pain 113:148-154.

Lynn B (1996) Efferent function of nociceptors. In: Neurobiology of nociceptors (Belmonte C, Cervero F, eds), pp 418-438. Oxford: Oxford UP.

Magerl W, Handwerker H (1988) A reliable model of experimental itching by iontophoresis of histamine. In: Proceedings of the Vth World Congress on Pain (Dubner R, Gebhart GF, Bond MR, eds), pp 536-540. Amsterdam: Elsevier Science.

Magerl W, Westerman RA, Möhner B, Handwerker HO (1990) Properties of transdermal histamine iontophoresis: differential effects of season, gender, and body region. J Invest Dermatol 94:347-352.
Masood K, Green BG, LaMotte RH (2005) Psychophysical measurements of pruritic and nociceptive sensations and dysesthetic states evoked by cutaneous application of cowhage spicules in human. Soc Neurosci Abstr 31:50.9.

Meyer RA, Campbell JN (1981) Myelinated nociceptive afferents account for the hyperalgesia that follows a burn to the hand. Science 213:1527-1529.

Polydefkis M, Hauer P, Sheth S, Sirdofsky M, Griffin JW, McArthur JC (2004) The time course of epidermal nerve fibre regeneration: studies in normal controls and in people with diabetes, with and without neuropathy. Brain 127:1606-1615.

Schmelz M, Schmidt R, Bickel A, Handwerker HO, Torebjörk HE (1997) Specific C-receptors for itch in human skin. J Neurosci 17:8003-8008.

Schmelz M, Michael K, Weidner C, Schmidt R, Torebörk HE, Handwerker HO (2000a) Which nerve fibers mediate the axon reflex flare in human skin? NeuroReport 11:645-648.

Schmelz M, Schmid R, Handwerker HO, Torebjork HE (2000b) Encoding of burning pain from capsaicin-treated human skin in two categories of unmyelinated nerve fibres. Brain 123:560-571.

Schmidt R, Schmelz M, Ringkamp M, Handwerker HO, Torebjork HE (1997) Innervation territories of mechanically activated C nociceptor units in human skin. J Neurophysiol 78:2641-2648.

Schmidt R, Schmelz M, Weidner C, Handwerker HO, Torebjörk HE (2002) Innervation territories of mechano-insensitive $\mathrm{C}$ nociceptors in human skin. J Neurophysiol 88:1859-1866.

Shelley WB, Arthur RP (1955a) Mucunain, the active pruritogenic proteinase of cowhage. Science 122:469-470.

Shelley WB, Arthur RP (1955b) Studies on cowhage (Mucuna pruriens) and its pruritogenic proteinase, mucunain. Arch Dermatol 72:399-406.

Shelley WB, Arthur RP (1957) The neurohistology and neurophysiology of the itch sensation in man. Arch Dermatol 76:296-323.

Simone DA, Ngeow JY, Whitehouse J, Becerra-Cabal L, Putterman GJ, LaMotte RH (1987) The magnitude and duration of itch produced by intracutaneous injections of histamine. Somatosens Res 5:81-92.

Simone DA, Alreja M, LaMotte RH (1991) Psychophysical studies of the itch sensation and itchy skin ("Alloknesis") produced by intracutaneous injection of histamine. Somatosens Mot Res 8:271-279.

Steinhoff M, Neisius U, Ikoma A, Fartasch M, Heyer G, Skov PS, Luger TA, Schmelz M (2003) Proteinase-activated receptor-2 mediates itch: a novel pathway for pruritus in human skin. J Neurosci 23:6176-6180.

Stevens SS, Galanter EH (1957) Ratio scales and category scales for a dozen perceptual continua. J Exp Psychol 54:377-411.

Toth-Kasa I, Jancso G, Bognar A, Husz S, Obal Jr F (1986) Capsaicin prevents histamine-induced itching. Int J Clin Pharmacol Res 6:163-169.

Tuckett RP, Wei JY (1987) Response to an itch-producing substance in cat. II. Cutaneous receptor populations with unmyelinated axons. Brain Res 413:95-103.

Twycross R, Greaves MW, Handwerker H, Jones EA, Libretto SE, Szepietowski JC, Zylicz Z (2003) Itch: scratching more than the surface. QJM 96:7-26.

van der Goot H, Timmerman H (2000) Selective ligands as tools to study histamine receptors. Eur J Med Chem 35:5-20.

von Frey M (1922) Zur physiologie der juckempfindung. Arc Néerl Physiol 7:142-145.

Weidner C, Schmelz M, Schmidt R, Hansson B, Handwerker HO, Torebjork HE (1999) Functional attributes discriminating mechano-insensitive and mechano-responsive $\mathrm{C}$ nociceptors in human skin. J Neurosci 19:10184-10190.

Weisshaar E, Heyer G, Forster C, Handwerker HO (1998) Effect of topical capsaicin on the cutaneous reactions and itching to histamine in atopic eczema compared to healthy skin. Arch Dermatol Res 290:306-311. 